\title{
Frequentist analysis of the parameter space of minimal supergravity
}

\author{
O. Buchmueller ${ }^{1}$, R. Cavanaugh ${ }^{2,3}$, D. Colling ${ }^{1}$, A. De Roeck ${ }^{4,5}$, M.J. Dolan ${ }^{6}$, J.R. Ellis ${ }^{4,7}$, H. Flächer ${ }^{8}$, \\ S. Heinemeyer ${ }^{9}$, K.A. Olive ${ }^{10, a}$, S. Rogerson ${ }^{1}$, F.J. Ronga ${ }^{11}$, G. Weiglein ${ }^{12}$ \\ ${ }^{1}$ High Energy Physics Group, Blackett Laboratory, Imperial College, Prince Consort Road, London SW7 2AZ, UK \\ ${ }^{2}$ Fermi National Accelerator Laboratory, P.O. Box 500, Batavia, IL 60510, USA \\ ${ }^{3}$ Physics Department, University of Illinois at Chicago, Chicago, IL 60607-7059, USA \\ ${ }^{4}$ CERN, 1211 Genève 23, Switzerland \\ ${ }^{5}$ Antwerp University, 2610 Wilrijk, Belgium \\ ${ }^{6}$ Institute for Particle Physics Phenomenology, University of Durham, South Road, Durham DH1 3LE, UK \\ ${ }^{7}$ Theoretical Physics and Cosmology Group, Department of Physics, King's College London, London WC2R 2LS, UK \\ ${ }^{8}$ Department of Physics and Astronomy, University of Rochester, Rochester, NY 14627, USA \\ ${ }^{9}$ Instituto de Física de Cantabria (CSIC-UC), 39005 Santander, Spain \\ ${ }^{10}$ William I. Fine Theoretical Physics Institute, University of Minnesota, Minneapolis, MN 55455, USA \\ ${ }^{11}$ Institute for Particle Physics, ETH Zürich, 8093 Zürich, Switzerland \\ ${ }^{12}$ DESY, Notkestrasse 85, 22607 Hamburg, Germany
}

Received: 7 December 2010 / Published online: 2 March 2011

(C) The Atuhor(s) 2011. This article is published with open access at Springerlink.com

\begin{abstract}
We make a frequentist analysis of the parameter space of minimal supergravity (mSUGRA), in which, as well as the gaugino and scalar soft supersymmetry-breaking parameters being universal, there is a specific relation between the trilinear, bilinear and scalar supersymmetrybreaking parameters, $A_{0}=B_{0}+m_{0}$, and the gravitino mass is fixed by $m_{3 / 2}=m_{0}$. We also consider a more general model, in which the gravitino mass constraint is relaxed (the VCMSSM). We combine in the global likelihood function the experimental constraints from low-energy electroweak precision data, the anomalous magnetic moment of the muon, the lightest Higgs boson mass $M_{h}, B$ physics and the astrophysical cold dark matter density, assuming that the lightest supersymmetric particle (LSP) is a neutralino. In the VCMSSM, we find a preference for values of $m_{1 / 2}$ and $m_{0}$ similar to those found previously in frequentist analyses of the constrained MSSM (CMSSM) and a model with common non-universal Higgs masses (NUHM1). On the other hand, in mSUGRA we find two preferred regions: one with larger values of both $m_{1 / 2}$ and $m_{0}$ than in the VCMSSM, and one with large $m_{0}$ but small $m_{1 / 2}$. We compare the probabilities of the frequentist fits in mSUGRA, the VCMSSM, the CMSSM and the NUHM1: the probability that mSUGRA is consistent with the present data is significantly less than in the other models. We also discuss the mSUGRA and
\end{abstract}

a e-mail: olive@physics.umn.edu
VCMSSM predictions for sparticle masses and other observables, identifying potential signatures at the LHC and elsewhere.

\section{Introduction}

One of the most favoured possible extensions of the Standard Model (SM) is supersymmetry (SUSY), which renders natural the electroweak mass scale [1-3] and accommodates grand unification of the particle interactions [4-7]. If $R$ parity is conserved it also provides a promising candidate for astrophysical cold dark matter, which might be the lightest neutralino, $\tilde{\chi}_{1}^{0}[8,9]$, or the gravitino [8-18]. SUSY also predicts the appearance of a relatively light Higgs boson [19-28], and may provide a welcome correction to the SM prediction for the anomalous magnetic moment of the muon, $(g-2)_{\mu}$ [29-38].

However, even the minimal supersymmetric extension of the SM, the MSSM [39], boasts over 100 free parameters, mostly associated with the mechanism of soft SUSYbreaking. Hence simplified scenarios with particular restrictions on the pattern of SUSY-breaking are often studied. One example is the constrained MSSM (CMSSM) [40-63], in which the soft SUSY-breaking gaugino masses $m_{1 / 2}$, scalar masses $m_{0}$ and trilinear couplings $A_{0}$ are each assumed to be universal at the grand unification (GUT) scale, and $\tan \beta$ 
is unconstrained. This leads to four effectively-free parameters, if the gravitino is assumed to be sufficiently heavy and/or rare that its cosmological decays and its mass are irrelevant. Another possibility is to relax the universality constraint for common soft SUSY-breaking contributions to the Higgs masses, yielding the NUHM1 [64-66] with five effective parameters in addition to the gravitino mass that is assumed to be irrelevant.

Alternatively, additional assumptions may be imposed, as in minimal supergravity (mSUGRA) [67-73], in which there is a specific relation between the trilinear and bilinear soft SUSY-breaking parameters and the universal scalar mass: $A_{0}=B_{0}+m_{0}$, and the gravitino mass is set equal to the common scalar mass before renormalization: $m_{3 / 2}=m_{0} .{ }^{1}$ Hence mSUGRA has just 3 free parameters, namely $m_{1 / 2}, m_{0}$ and $A_{0}$, and $\tan \beta$ is now fixed by the radiative electroweak symmetry breaking conditions [74-77]. Further, there is a restriction on $m_{0}$ if the lightest supersymmetric particle (LSP) is the lightest neutralino, $\tilde{\chi}_{1}^{0}$, as we assume here for the reasons discussed below. An intermediate scenario is the very constrained MSSM (VCMSSM), in which again $A_{0}=B_{0}+m_{0}$ but the gravitino mass is left free, so there is no restriction on $m_{0}[78,79]$. If $m_{3 / 2}$ is sufficiently large, and/or the gravitino abundance is sufficiently low (as we will assume here), there are no related cosmological constraints, and the VCMSSM also has effectively 3 relevant free parameters, but they are less constrained than in mSUGRA.

We have previously published frequentist analyses of the CMSSM and NUHM1 parameter spaces [80-83], ${ }^{2}$ implementing the experimental constraints from low-energy electroweak precision data and the lightest Higgs boson mass $M_{h}$ as well as the lower limits from the direct searches for SUSY particles at LEP, fitting the measured value of the anomalous magnetic moment of the muon, $(g-2)_{\mu}$, $B$ physics and the cosmological dark matter density, $\Omega_{\chi} h^{2}$, assuming that the LSP is the lightest neutralino, $\tilde{\chi}_{1}^{0}$. In this paper we extend these analyses to include the VCMSSM and mSUGRA which have, as discussed above, one or two additional constraints on the pattern of soft SUSY-breaking, respectively. An early $\chi^{2}$ analysis in these scenarios can be found in [63].

In the case of the VCMSSM, in which $A_{0}=B_{0}+m_{0}$ but the gravitino mass is free, we assume that the gravitino is sufficiently heavy or rare that the dark matter is composed of neutralinos and the cosmological effects of its decays are unimportant. Under these assumptions, as we show below, imposing the neutralino dark matter constraint does not increase substantially the $\chi^{2}$ of the global minimum, which is $\sim 1.2$ higher than in the CMSSM, but we find 68 and $95 \%$

\footnotetext{
${ }^{1}$ See, for example, remark (b) following (16) of [70].

${ }^{2}$ See: http://cern.ch/mastercode.
}

confidence-level (CL) ranges of $m_{1 / 2}$ and $m_{0}$ that are more restrictive than those found previously in our analyses of the CMSSM and the NUHM1.

However, the preferred part of the VCMSSM parameter space has $m_{\tilde{\chi}_{1}^{0}}>m_{0}$, which within mSUGRA would imply that $m_{3 / 2}=m_{0}<m_{\tilde{\chi}_{1}^{0}}$, so that the lightest neutralino would be unstable, and the dark matter would be composed of gravitinos. In such a case, the usual calculation of the neutralino dark matter density $\Omega_{\chi} h^{2}$ would be inapplicable, and one should, instead, consider the constraints on the decays of long-lived neutralinos or other sparticles into gravitinos that are imposed by the cosmological abundances of light elements [16-18, 84-89]. In fact, these constraints are sufficiently strong to exclude, within mSUGRA and assuming a standard cosmological evolution, the otherwise preferred regions of parameter space with $m_{0}=m_{3 / 2}<m_{\tilde{\chi}_{1}^{0}}$ and hence gravitino dark matter [84]. ${ }^{3}$ Thus, the surviving region of the mSUGRA parameter space has $m_{3 / 2}=m_{0}>m_{\tilde{\chi}_{1}^{0}}$, corresponding to neutralino dark matter.

We find within the mSUGRA model two distinct regions with local minima of the $\chi^{2}$ function, each of which is significantly worse than in the VCMSSM, namely $\Delta \chi^{2} \sim 7$ or 11 with 19 dof, corresponding to a goodness-of-fit of just $6.0 \%$ (or $2.3 \%$ ) in the mSUGRA hypothesis. This may be contrasted with the cases of the CMSSM and the NUHM1 studied in [80-83], where the best-fit parameters are consistent with the current experimental constraints at the level of $32 \%(31 \%)$ fit probability, and with the VCMSSM case ( 20 dof, $31 \%$ fit probability). One of the regions preferred in mSUGRA has larger values of both $m_{1 / 2}$ and $m_{0}$ than in the VCMSSM, CMSSM and NUHM1, and the other has larger $m_{0}$ but small $m_{1 / 2}$.

\section{Notations}

Before describing our analyses of the VCMSSM and mSUGRA in more detail, we first specify our notations, since different conventions for the MSSM superpotential couplings and the trilinear and bilinear soft SUSYbreaking terms are used elsewhere in the literature, including in [80]. Our conventions here follow those specified, e.g., in [91], according to which the superpotential includes the terms

$$
\begin{aligned}
W \ni & Y_{e} \epsilon H_{1} L E^{c}+Y_{d} \epsilon H_{1} Q D^{c} \\
& +Y_{u} \epsilon H_{2} Q U^{c}+\mu \epsilon H_{1} H_{2},
\end{aligned}
$$

\footnotetext{
${ }^{3}$ See [90] for possible effects of non-standard cosmological histories that might invalidate these arguments against the gravitino LSP scenario.
} 
where the $Y_{i}$ are Yukawa couplings, $\epsilon$ is the antisymmetric $2 \times 2$ tensor with $\epsilon_{12}=+1, H_{1,2}, L, E^{c}, Q, D^{c}$ and $U^{c}$ are superfields, and $\mu$ is the Higgs supermultiplet mixing parameter. The corresponding trilinear and bilinear SUSY-breaking terms in the effective Lagrangian are:

$\mathcal{L} \ni-\left(A_{t} Y_{t} \epsilon h_{2} q t^{c}+\cdots\right)-\mu B \epsilon h_{1} h_{2}$,

where the lower-case letters denote the scalar components of the corresponding superfields. Within this convention, $\sin 2 \beta=-2 B \mu /\left(m_{1}^{2}+m_{2}^{2}+2 \mu^{2}\right)$ at the tree level, where $m_{1,2}$ is the soft SUSY-breaking mass of $H_{1,2}$, the left-right mixing term in the stop mixing matrix is $m_{\mathrm{LR}}^{2}=-m_{t}\left(A_{t}+\right.$ $\mu \cot \beta$ ), and the one-loop renormalization of the trilinear coefficient has the form $d A_{t} / d t \ni-\frac{16}{3} g_{3}^{2} M_{3}+6 Y_{t}^{2} A_{t}+\cdots$ (where $M_{3}$ denotes the soft SUSY-breaking parameter in the gluino sector, and $A_{t}$ is the trilinear Higgs-stop coupling). These choices unambiguously determine the sign conventions for $A$ and $B$.

Within this convention, $A_{0}=B_{0}+m_{0}$ before renormalization in mSUGRA with its minimal (flat) Kähler potential. ${ }^{4}$ Additionally, as already mentioned, the choice of a minimal Kähler potential also imposes the condition $m_{3 / 2}=$ $m_{0}$ on the gravitino mass before renormalization, so that mSUGRA has just three independent parameters.

\section{Details of the evaluation}

Our analysis has been performed using the MasterCode [80-83]. ${ }^{5}$ We sample the VCMSSM and mSUGRA parameter spaces using a Markov Chain Monte Carlo (MCMC) technique similar to that used in our previous analyses of the CMSSM and NUHM1 [80-83]. We evaluate the global likelihood using a $\chi^{2}$ function constructed by combining the likelihoods for the experimental constraints from electroweak precision data, the anomalous magnetic moment of the muon, $(g-2)_{\mu}, B$ physics, the astrophysical cold dark matter density, $\Omega_{\chi} h^{2}$, and searches for the lightest Higgs boson and supersymmetric particles, in exactly the same way as described previously [80-82]. The most significant change in our numerical treatments of these observables since [82] is in $(g-2)_{\mu}$, for which we use the estimate $a_{\mu}^{\mathrm{SUSY}}=(28.7 \pm 8.0) \times 10^{-10}[38]$.

The numerical evaluation within the MasterCode combines the following theoretical codes. For the RGE running of the soft SUSY-breaking parameters, it uses

\footnotetext{
${ }^{4}$ Note that many of the publicly available packages such as SoftSUSY [92] use the opposite sign convention, as may be ascertained by comparing the signs of the gauge and Yukawa contributions to the RGEs of the $A$ parameters. In the notation of these codes, the mSUGRA boundary condition would be $A_{0}=B_{0}-m_{0}$.

${ }^{5}$ See footnote 2 .
}

SoftSUSY [92], which is combined consistently with the codes used for the low-energy observables: FeynHiggs [28, 93-95] is used for the evaluation of the Higgs masses and $a_{\mu}^{\text {SUSY }}$ (see also [96-99]). For flavour-related observables we use SuFla [100, 101] and SuperIso [102, 103], and for the electroweak precision data we have included a code based on [104, 105]. Finally, for dark-matter-related observables, we use MicrOMEGAs [106-108] and DarkSUSY $[109,110]$. We make extensive use of the SUSY Les Houches Accord [111,112] in the combination of the various codes within the MasterCode.

Our MCMC sampling of the VCMSSM parameter space comprises some $30,000,000$ points. The neutralino CDM constraint on $\Omega_{\chi} h^{2}$ [113] and the Higgs mass constraint $[114,115]$ were applied after the sampling, allowing the effects of these two constraints to be studied separately. In the case of mSUGRA, about 17,000,000 of the MCMC points from the VCMSSM sample survive the mSUGRA constraint $m_{0}=m_{3 / 2}>m_{\tilde{\chi}_{1}^{0}}$, and we again applied the neutralino CDM constraint on $\Omega_{\chi} h^{2}$ and the Higgs mass constraint a posteriori.

\section{Analysis of parameter planes}

We start our analysis with the results in the $\left(m_{0}, m_{1 / 2}\right)$ planes. Figure 1 displays the global likelihood functions in the VCMSSM (left panels) and mSUGRA (right panels). In each case, the upper panel shows results before the $\Omega_{\chi} h^{2}$ constraint is applied, and the lower panel displays the effects of imposing the $\Omega_{\chi} h^{2}$ constraint. ${ }^{6}$ In all panels, we display the points with the minimal values of $\chi^{2}$ (green stars) as well as the 68 and $95 \%$ CL contours (red and blue), corresponding to $\Delta \chi^{2}=2.28$ and 5.99. Other contours of $\Delta \chi^{2}$ are indicated in shades of grey.

In the upper left panel for the VCMSSM before applying the $\Omega_{\chi} h^{2}$ constraint, the triangular region at small $m_{0}$ and large $m_{1 / 2}$ is excluded because there the LSP would be charged, and a band extending to large $m_{0}$ at low $m_{1 / 2}$ is excluded by the LEP Higgs constraint. The best-fit point is at $\left(m_{0}, m_{1 / 2}\right)=(30,310) \mathrm{GeV}$, the 68 and $95 \% \mathrm{CL}$ contours enclose regions of the $\left(m_{0}, m_{1 / 2}\right)$ planes that are similar to those favoured in the CMSSM and NUHM1 [81, 82], and there are no preferred points in the focus-point region at large $m_{0}$. Specifically, we find that the focus-point region at large $m_{0} \sim 2000 \mathrm{GeV}$ with $m_{1 / 2} \sim 200 \mathrm{GeV}$ is subject to a penalty of $\Delta \chi^{2} \sim 10$.

\footnotetext{
${ }^{6}$ We recall that the constraints due to the late decays of massive metastable particles [84] are taken into account implicitly as described above, i.e., in the VCMSSM by assuming that the gravitino mass is high and/or its primordial density is low, and by accepting that the decay constraints are so severe in mSUGRA as to forbid $m_{3 / 2}=m_{0}<$ $m_{\tilde{\chi}_{1}^{0}}$ in that case.
} 
In the upper right panel for mSUGRA without the $\Omega_{\chi} h^{2}$ constraint, we see a similar pattern, but with a larger excluded triangular region at large $m_{1 / 2}$ and small $m_{0}$, as the allowed part of the $\left(m_{0}, m_{1 / 2}\right)$ plane is now restricted to the region where $m_{3 / 2}=m_{0}>m_{\tilde{\chi}_{1}^{0}} \sim 0.4 m_{1 / 2}$. Since the bestfit VCMSSM point seen in the upper left panel of Fig. 1 lies in the region that is disallowed in mSUGRA, there is a new mSUGRA best-fit point on the boundary of the allowed region, with $\left(m_{0}, m_{1 / 2}\right)=(110,280) \mathrm{GeV}$. The minimum value of $\chi^{2}$ is higher than in the VCMSSM model by $\sim 1.5$, and the $\Delta \chi^{2}$ values in other regions of the $\left(m_{0}, m_{1 / 2}\right)$ plane are correspondingly reduced, leading to the emergence of 'archipelago' of points at $\left(m_{0}, m_{1 / 2}\right) \sim(700,1000) \mathrm{GeV}$ that are now allowed at the $95 \% \mathrm{CL}$. The fact that the mSUGRA best-fit point lies on the boundary of the allowed parameter space indicates that the restrictions in this model are disfavoured by current experimental data.

We recall that it was shown in [81] that in the CMSSM the $\Omega_{\chi} h^{2}$ constraint [113] has a relatively modest impact on the preferred ranges in the $\left(m_{0}, m_{1 / 2}\right)$ plane. However, imposing the $\Omega_{\chi} h^{2}$ constraint has a dramatic effect on the VCMSSM fit, as we see in the lower left panel of Fig. 1. The region allowed at the $95 \% \mathrm{CL}$ is reduced to a narrow 'WMAP strip' terminating at $\left(m_{0}, m_{1 / 2}\right) \sim(250,700) \mathrm{GeV}$. We recall that similar WMAP coannihilation strips appear in the CMSSM for fixed values of $\tan \beta$ and $A_{0}$, but move across the $\left(m_{0}, m_{1 / 2}\right)$ plane as $\tan \beta$ and $A_{0}$ are varied, which was why the WMAP strip structure was invisible in the global likelihood fit to the CMSSM [81]. On the other hand, we recall that, in the VCMSSM, $\tan \beta$ is fixed as a function of $m_{0}, m_{1 / 2}$ and $A_{0}$, and the dependence on $A_{0}$ is not very strong. As a result of the loss of the freedom to vary $\tan \beta$ independently, the WMAP strip structure is resurrected in the VCMSSM.

The best-fit point in the VCMSSM with the $\Omega_{\chi} h^{2}$ constraint has $\left(m_{0}, m_{1 / 2}, A_{0}\right) \sim(60,300,30) \mathrm{GeV}$ and $\tan \beta \sim 9$. However, as in the previous CMSSM and NUHM1 cases, rather larger values of $m_{1 / 2}$, and hence $m_{\tilde{\chi}_{1}^{0}}, m_{\tilde{g}}$ and other sparticle masses, are allowed at the $95 \% \mathrm{CL}$. This VCMSSM fit has a very good value of $\chi^{2} /$ dof $=22.5 / 20$
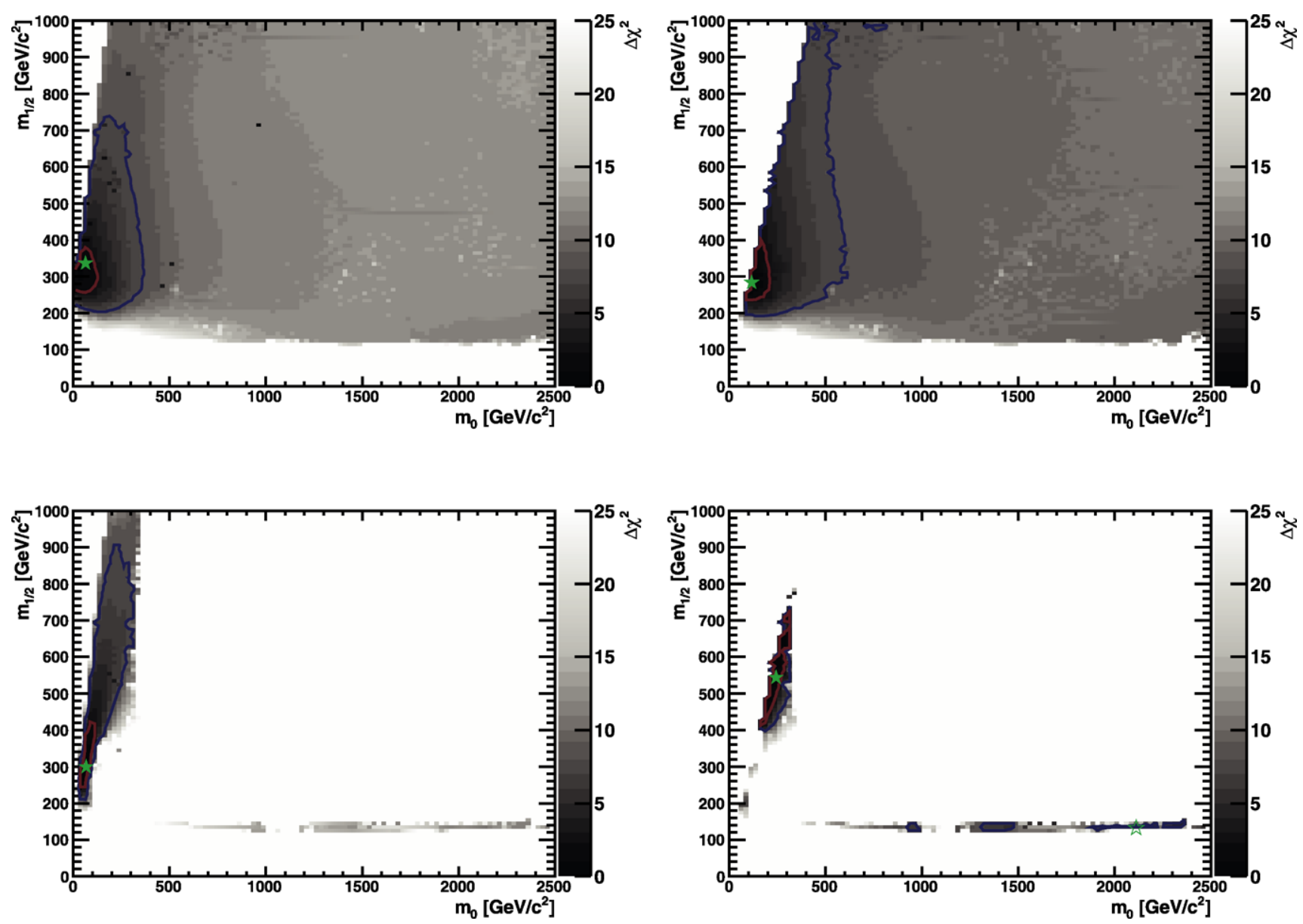

Fig. 1 (Color online) The $\left(m_{0}, m_{1 / 2}\right)$ planes in the VCMSSM (left panels) and mSUGRA (right panels), without (upper) and with (lower panels) the $\Omega_{\chi} h^{2}$ constraint [113], showing in each case the best-fit

points (green stars) and the 68 and 95\% CL contours (red and black, respectively). The open green star in the lower right panel denotes the secondary minimum discussed in the text 
( $31 \%$ probability), similar to the $\chi^{2}$ before applying the $\Omega_{\chi} h^{2}$ constraint, demonstrating that there is no significant tension between this and other constraints. The increase in $\chi^{2}$ due to inclusion of the LEP $M_{h}$ constraint $[114,115]$ is $\sim 1.1$, demonstrating that there is also no significant tension between the $M_{h}$ and other constraints. Moreover, the fact that the fit probability in the VCMSSM is about the same as the value of $32 \%\left(\chi^{2}=21.3 / 19\right.$ dof $)$ found in the CMSSM indicates that applying the extra VCMSSM constraint $A_{0}=B_{0}+m_{0}$ is certainly not a source of significant tension in the fit.

We note that there is no focus-point region visible in the VCMSSM when the $\Omega_{\chi} h^{2}$ constraint is applied, since it is not compatible with the $\tan \beta$ constraint imposed by the initial conditions. On the other hand, there is a very narrow strip at $m_{1 / 2} \sim 130 \mathrm{GeV}$ extending to large $m_{0}$, where the relic density is brought into the WMAP range by rapid annihilation through the direct-channel light Higgs pole. However, this strip has $\Delta \chi^{2} \geq 9$.

Turning finally to the lower right panel of Fig. 1 for mSUGRA with the $\Omega_{\chi} h^{2}$ constraint applied, we see an evolution of the picture. Much of the VCMSSM 'WMAP strip' has disappeared, as only a vestige of it has $m_{\tilde{\chi}_{1}^{0}}<m_{0}=$ $m_{3 / 2}$. Since the minimum value of $\chi^{2}$ in the VCMSSM was located in the forbidden region with $m_{\tilde{\chi}_{1}^{0}}>m_{0}=m_{3 / 2}$, the minimum value of $\chi^{2}$ in the mSUGRA region is significantly higher, specifically $\chi^{2} \sim 29$, a price $\Delta \chi^{2} \sim 7$. The best mSUGRA fit is again on the boundary of the allowed region. Moreover, as indicated by the open green star in the lower right panel of Fig. 1, a second local minimum with $\chi^{2} \sim 33$ (which is therefore allowed at the 95\% CL) can be found along the light Higgs rapid-annihilation strip with $m_{1 / 2} \sim 130 \mathrm{GeV}$ and $900 \mathrm{GeV} \lesssim m_{0} \lesssim 2500 \mathrm{GeV}$. Along this strip, the $\chi^{2}$ function is relatively insensitive to $m_{0}$, thanks to approximate compensation between the contributions from $\mathrm{BR}(b \rightarrow s \gamma),(g-2)_{\mu}$ and the forward-backward asymmetry of $b$ quarks measured at LEP, decreasing slightly as $m_{0}$ increases up to $m_{0} \lesssim 2100 \mathrm{GeV}$.

Figure 2 displays the $\left(\tan \beta, m_{1 / 2}\right)$ planes in the VCMSSM (left) and mSUGRA (right, where the cut $m_{\tilde{\chi}_{1}^{0}}<m_{0}$ was applied), both without (upper) and with (lower) the $\Omega_{\chi} h^{2}$ constraint. In the upper left panel, we see that a range of $\tan \beta \sim 6$ to $12-$ similar to that favoured in
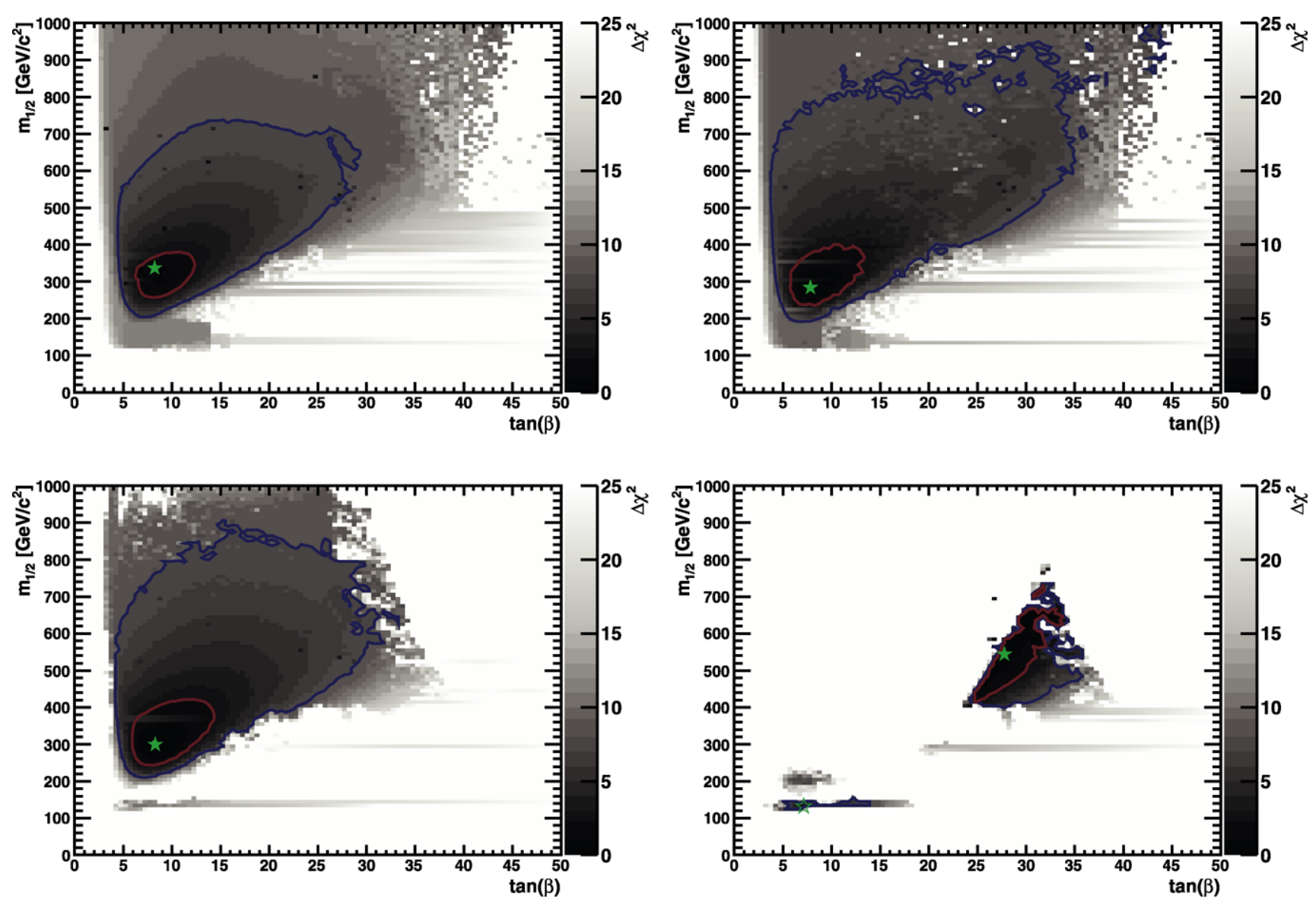

Fig. 2 The $\left(\tan \beta, m_{1 / 2}\right)$ planes in the VCMSSM (left) and mSUGRA (right), without (upper) and with (lower panels) the $\Omega_{\chi} h^{2}$ constraint [113], showing in each case the best-fit points and the 68 and 95\% CL contours 

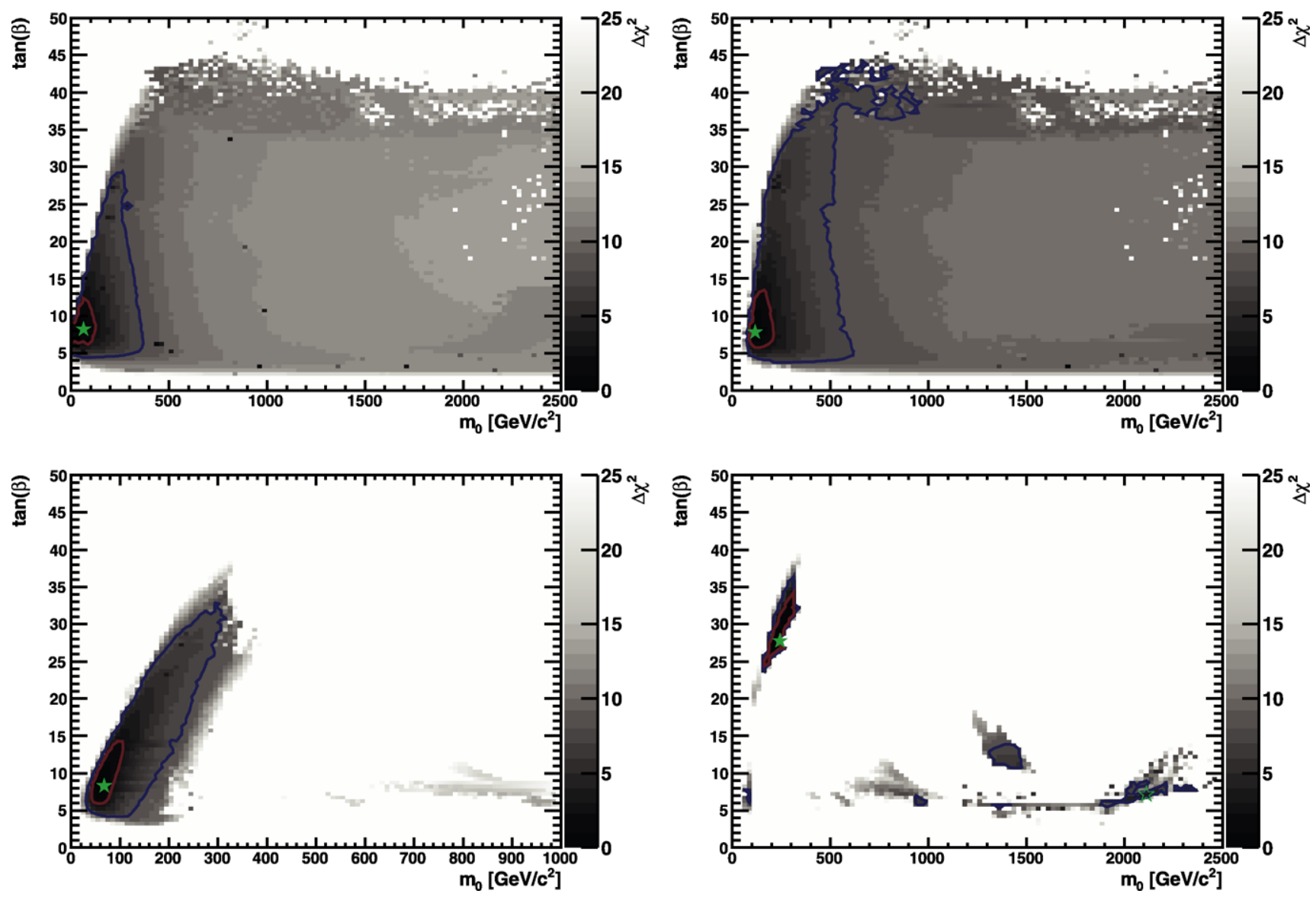

Fig. 3 The $\left(m_{0}, \tan \beta\right)$ planes in the VCMSSM (left) and mSUGRA (right), without (upper) and with (lower panels) the $\Omega_{\chi} h^{2}$ constraint [113], showing in each case the best-fit points and the 68 and 95\% CL contours

the CMSSM and NUHM1-is preferred in the VCMSSM at the $68 \% \mathrm{CL}$, but with a best-fit value $\tan \beta \sim 9$ that is rather smaller. A range of slightly larger $\tan \beta \sim 6$ to 13 is allowed in mSUGRA at the $68 \% \mathrm{CL}$, with a best-fit value of $\tan \beta \sim 8$ before imposing the $\Omega_{\chi} h^{2}$ constraint. The $95 \%$ CL ranges of $\tan \beta$ extend to $\sim 30,35$ in the VCMSSM and mSUGRA, respectively. When the $\Omega_{\chi} h^{2}$ constraint is imposed on the VCMSSM (lower left), the ranges of $\tan \beta$ favoured at the 68 and $95 \% \mathrm{CL}$ are little changed, with $\tan \beta \sim 9$ at the best-fit point. We again see at higher $\Delta \chi^{2}$ the rapid-annihilation Higgs funnel at $m_{1 / 2} \sim 130 \mathrm{GeV}$, separated from the favoured coannihilation region. However, when the $\Omega_{\chi} h^{2}$ constraint is imposed on mSUGRA (lower right), the coannihilation region shrinks to a vestigial region with $\tan \beta \sim 30$ and relatively high $\chi^{2}$, as previously remarked, and the other minimum along the light Higgs funnel has $\Delta \chi^{2} \sim 4$ and $\tan \beta \sim 5$ to 14 , and also contains an area allowed at the $95 \%$ CL.

Figure 3 displays the corresponding $\left(m_{0}, \tan \beta\right)$ planes in the VCMSSM (left) and mSUGRA (right). In the absence of the $\Omega_{\chi} h^{2}$ constraint (upper panels), we see regions allowed at the $95 \% \mathrm{CL}$ that are restricted to $m_{0} \sim 300 \mathrm{GeV}$ in the
VCMSSM case (left) and $\sim 500 \mathrm{GeV}(\sim 1000 \mathrm{GeV}$ including the 'archipelago') in the mSUGRA model (right), the larger range being expected from the restriction $m_{0}>m_{\tilde{\chi}_{1}^{0}}$. Imposing the $\Omega_{\chi} h^{2}$ constraint on the VCMSSM (lower left), we find a band at low $m_{0}$ that can be identified with the WMAP-compatible coannihilation strip in the corresponding panel of Fig. 1. Turning to the case of mSUGRA with the $\Omega_{\chi} h^{2}$ constraint (lower right), only a vestige of the low$m_{0}$ band remains at relatively large $\tan \beta$, and the $\chi^{2}$ in this region is $\sim 4$ smaller than in the rapid-annihilation region at larger $m_{0}$ and smaller $\tan \beta$ values. $^{7}$

Finally, Fig. 4 displays the $\left(A_{0} / m_{0}, \tan \beta\right)$ planes for the VCMSSM (left) and mSUGRA (right). In the VCMSSM, a wide range of values extending from $A_{0} / m_{0} \sim+2$ down to $\sim-3$ (corresponding to the case of very small $m_{0}$ ) lies

\footnotetext{
${ }^{7}$ Because the rapid-annihilation region is very narrow in $m_{1 / 2}$, and quite broad in $m_{0}$ and $A_{0}$, it is difficult to sample fully using the MCMC approach, even with the high statistics of our full sample. Moreover, the MCMC approach samples the input variables $m_{1 / 2}, m_{0}$ and $A_{0}$, contributing also to the uneven sampling in the derived quantity $\tan \beta$ seen in the lower panels of Fig. 3 .
} 

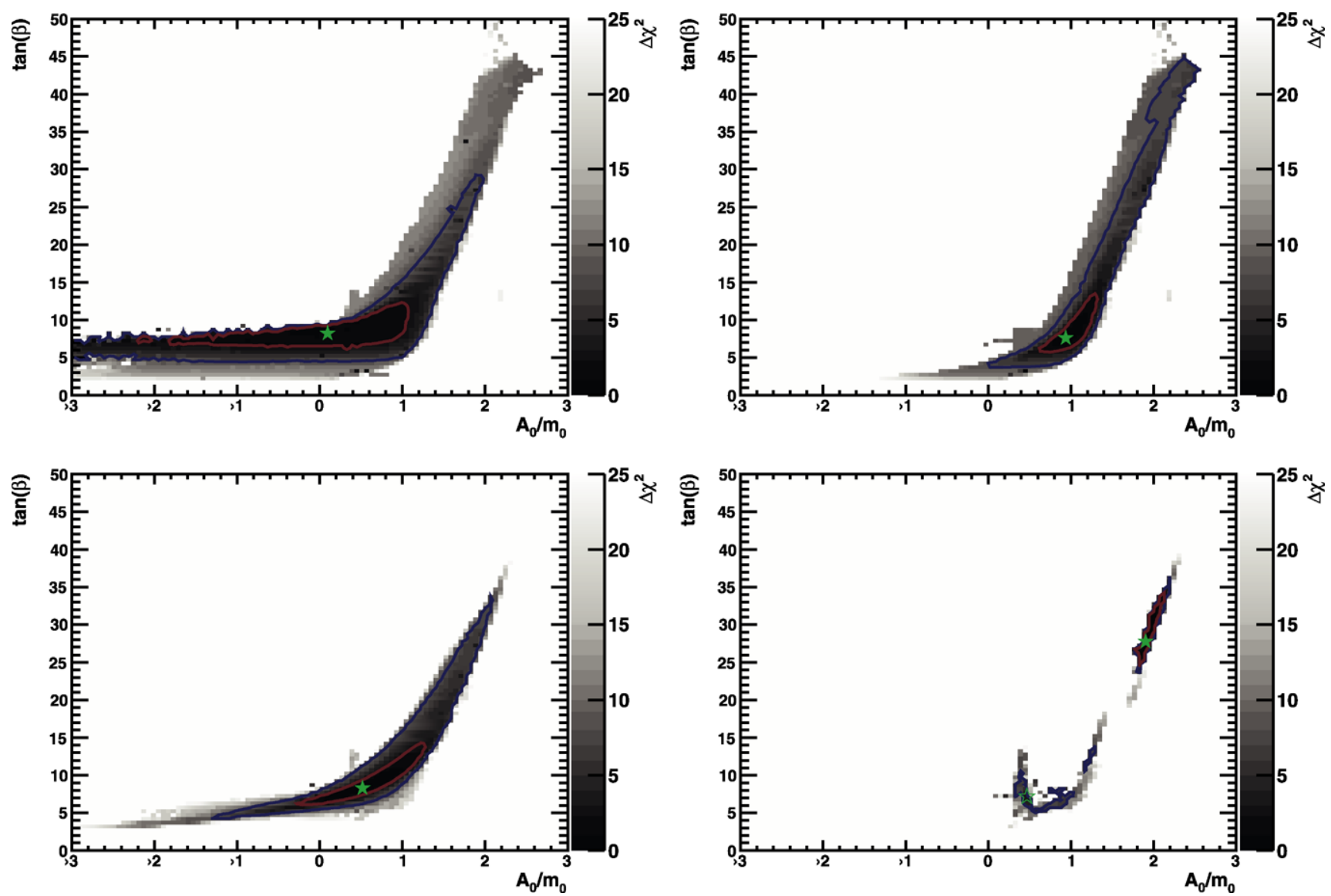

Fig. 4 The $\left(A_{0} / m_{0}, \tan \beta\right)$ planes in the VCMSSM (left) and mSUGRA (right), without (upper) and with (lower panels) the $\Omega_{\chi} h^{2}$ constraint [113], showing the best-fit points and the 68 and $95 \%$ CL contours in each case

within the $95 \% \mathrm{CL}$ contour, whereas in mSUGRA only positive values are favoured at the 95\% CL before the $\Omega_{\chi} h^{2}$ constraint is applied. On the other hand, when it is applied, large negative values of $A_{0} / m_{0}$ are disfavoured also in the VCMSSM, and values of $A_{0} / m_{0} \sim 2$ with $\tan \beta \sim 30$ are allowed at the $95 \% \mathrm{CL}$. Two distinct populations of preferred points are apparent in mSUGRA when the $\Omega_{\chi} h^{2}$ constraint is applied. There is a vestigial coannihilation region with $A_{0} / m_{0} \sim 2$ and $\tan \beta \sim 28$, and the rapid-annihilation funnel region has $A_{0} / m_{0} \sim 0.4$ and $\tan \beta \sim 7$.

We recall that the simplest Polonyi model of SUSY breaking [67] predicts that $\left|A_{0} / m_{0}\right|=3-\sqrt{3}$, a possibility that is quite consistent with the VCMSSM both before and after applying the $\Omega_{\chi} h^{2}$ constraint (left panels), but only marginally consistent with the $95 \%$ CL region of mSUGRA.

\section{Comparison of likelihood analyses}

We gather in Table 1 some important aspects of the best-fit points in mSUGRA, the VCMSSM, the CMSSM and the NUHM1 (the latter being adapted from [81, 82], with the inclusion of the updated values of $(g-2)_{\mu}$ and $m_{t}$ : we find that the values of the different parameters at the best fit points in the CMSSM and the NUHM1, as well as the fit probabilities, remain essentially unchanged with respect to the analysis in $[81,82])$. In the case of mSUGRA, we list the properties of two local minima of the $\chi^{2}$ function: one is in the coannihilation region and one in the light Higgs rapidannihilation funnel with $\Delta \chi^{2} \sim 4$, as discussed earlier. We see in the first column that the mSUGRA fits have substantially higher $\chi^{2}$ than the other models, which is reflected in the second column by a significantly lower probability. The CMSSM and NUHM1 gave comparable fit probabilities of $32(31) \%$ and the VCMSSM fit has a probability of $31 \%$, while mSUGRA, with a probability of $6.0 \%$ in the coannihilation region and $2.3 \%$ in the light Higgs funnel region, provides a worse description of the data considered in this analysis. As a result, the mSUGRA scenario is somewhat disfavoured compared to the other SUSY scenarios we consider. The source of tension in mSUGRA is seen by comparing the third and fourth columns. The best VCMSSM fit has very similar values of $m_{1 / 2}$ and $m_{0}$ to those in the CMSSM, and in both cases $m_{0} \ll m_{1 / 2}$. The conflict between this preference and the cosmological requirement on 
Table 1 Comparison of the best fits found in this paper within the mSUGRA and VCMSSM frameworks with previous results [81, 82] in the CMSSM and NUHM1 frameworks. In addition to the minimum value of $\chi^{2}$ in each scenario, we include the values of $m_{1 / 2}, m_{0}, A_{0}$ and $\tan \beta$ at all the best-fit points, as well as $M_{h}$ (for the latter the direct bounds from LEP and the Tevatron are not included). All masses are in $\mathrm{GeV}$ units. We list two very different mSUGRA fit results with similar $\chi^{2}$ values: the first is in the coannihilation region, and the second is in the light Higgs funnel region. Note that we use here the convention for $A_{0}$ described in the text, which differs from that in [80]

\begin{tabular}{llllrrrr}
\hline Model & Minimum $\chi^{2}$ & Probability & $m_{1 / 2}$ & $m_{0}$ & $A_{0}$ & $\tan \beta$ & $M_{h}($ no LEP) \\
\hline mSUGRA & 29.4 & $6.0 \%$ & 550 & 230 & 430 & 28 & 107.7 \\
& 33.2 & $2.3 \%$ & 130 & 2110 & 980 & 7 & 116.9 \\
VCMSSM & 22.5 & $31 \%$ & 300 & 60 & 30 & 9 & 109.3 \\
CMSSM & 21.3 & $32 \%$ & 320 & 60 & -160 & 11 & 107.9 \\
NUHM1 & 19.3 & $31 \%$ & 260 & 100 & 1010 & 8 & 119.5 \\
\hline
\end{tabular}

mSUGRA that $m_{3 / 2}=m_{0}>m_{\tilde{\chi}_{1}^{0}}$ leads to a best fit in the coannihilation region with larger values of $m_{1 / 2}$ and $\tan \beta$ as well as a larger value of $m_{0}$, and the other local minimum with small $m_{1 / 2}$ and much larger $m_{0} .{ }^{8}$ As seen in the sixth column, the values of $\tan \beta$ favoured in the VCMSSM, CMSSM and NUHM1 are much smaller than that favoured at the best-fit point of mSUGRA in the coannihilation region. The last column shows the best-fit values of $M_{h}$, not taking into account the LEP and Tevatron limits. It can be seen that the best-fit values of $M_{h}$ in mSUGRA in the coannihilation region, the VCMSSM and the CMSSM are similar, and somewhat below the LEP lower limit, leading to increases in $\chi^{2}$ of 3.9 (1.1) (1.4) when the LEP constraint is applied. On the other hand, we find rather higher best-fit values of $M_{h}$ in the NUHM1 and mSUGRA in the funnel region. Finally, we note that the largest variations between the different models occur for $A_{0}$ (fifth column), reflecting the relative insensitivity of our fits to this parameter.

In Fig. 5 we display the spectra in the VCMSSM (top) and mSUGRA (middle and bottom) with the $\Omega_{\chi} h^{2}$ constraint applied, complementing the CMSSM and NUHM1 spectra shown in Fig. 3 of [82]. In the case of the VCMSSM, the spectrum is qualitatively similar to those in the CMSSM and NUHM1 $[81,82]$. The two mSUGRA spectra are in the coannihilation (middle) and funnel region (bottom), reflecting the coexistence of two qualitatively different (near-)bestfit points with relatively similar $\chi^{2}$. The spectra in these regions are significantly different from each other and from the VCMSSM, CMSSM and NUHM1. This is because the coannihilation region has $m_{1 / 2}$ significantly larger than in the other models, whereas the funnel region has a significantly smaller and very well-defined value of $m_{1 / 2}$ and rel-

\footnotetext{
${ }^{8}$ On the other hand, the best NUHM1 fit has a value of $m_{1 / 2}$ similar to those in the VCMSSM and CMSSM but a somewhat larger value of $m_{0}$, which is possible because an acceptable value of $\Omega_{\chi} h^{2}$ may be found along a funnel in the $\left(m_{0}, m_{1 / 2}\right)$ plane at lower values of $\tan \beta$, due to $\tilde{\chi}_{1}^{0} \tilde{\chi}_{1}^{0}$ annihilation through direct-channel $H, A$ poles. This option is not available in the CMSSM except at large $\tan \beta$ and $m_{1 / 2}$, and it is absent completely in mSUGRA.
}

atively large values of $m_{0}$. This bimodality affects directly the preferred values of $m_{\tilde{\chi}_{1}^{0}}$ and $m_{\tilde{g}}$, and affects the other sparticle masses via renormalization effects. These spectra show that the coloured particles are within the reach of the LHC for the VCMSSM and MSUGRA in the coannihiliation region, whereas more integrated luminosity would be necessary for mSUGRA in the funnel region (except for gluino production). In each scenario some SUSY particles should be accessible at an $e^{+} e^{-}$collider, even with a center-of-mass energy as low as $500 \mathrm{GeV}$.

We display in Fig. 6 the one-dimensional $\chi^{2}$ likelihood functions for (top panels) $m_{\tilde{g}}$ and $m_{\tilde{\chi}_{1}^{0}}$, (middle panels) the mass differences $m_{\tilde{q}_{R}}-m_{\tilde{g}}$ and $m_{\tilde{\tau}_{1}}-m_{\tilde{\chi}_{1}^{0}}$, and (bottom panels) $\operatorname{BR}\left(B_{s} \rightarrow \mu^{+} \mu^{-}\right)$and $\sigma_{p}^{\mathrm{SI}}$, the spin-independent neutralino scattering cross section. The NUHM1 curves are shown as purple dotted lines, the CMSSM curves as green dash-dotted lines, the VCMSSM curves as red dashed lines, and the mSUGRA curves as blue solid lines. In each plot, we display the $\Delta \chi^{2}$ contribution of each model relative to the best-fit point in that model. Thus the secondary minimum of the $\chi^{2}$ function for mSUGRA has the appropriate $\Delta \chi^{2}$ relative to the absolute minimum in that model.

We see that the likelihood functions for $m_{\tilde{g}}$ and $m_{\tilde{\chi}_{1}^{0}}$ in the VCMSSM are similar to those in the CMSSM and NUHM1 [81, 82], with the most likely range of $m_{\tilde{g}} \sim 700$ to $800 \mathrm{GeV}$ and $m_{\tilde{\chi}_{1}^{0}} \sim 120 \mathrm{GeV}$. The corresponding likelihood functions in mSUGRA, on the other hand, are very different, reflecting once more the bimodality in $m_{1 / 2}$ and $m_{0}$. In the VCMSSM, as in the CMSSM and NUHM1, the preferred range of $m_{\tilde{g}}$ (top left panel) suggests that there may be good prospects for observing first hints of SUSY at the LHC in 2011/2012, although values of $m_{\tilde{g}}>2000 \mathrm{GeV}$ are permitted in the NUHM1 with $\Delta \chi^{2} \sim 5$. In the rapidannihilation strip of mSUGRA, $m_{\tilde{g}} \sim 400 \mathrm{GeV}$ and $m_{\tilde{\chi}_{1}^{0}} \sim$ $55 \mathrm{GeV}$, putting the discovery of the gluino at the LHC within the reach of the LHC in 2011/2012, and making $m_{\tilde{g}}$ potentially a powerful diagnostic tool for mSUGRA. However, discovery of the gluino would be delayed in the 
Fig. 5 Spectra in the VCMSSM (top), and mSUGRA in the coannihilation region (middle) and the funnel region (bottom), implementing all the constraints including that on $\Omega_{\chi} h^{2}$. The horizontal solid lines indicate the best-fit values, the vertical solid lines are the $68 \% \mathrm{CL}$ ranges, and the vertical dashed lines are the $95 \%$ CL ranges for the indicated mass parameters
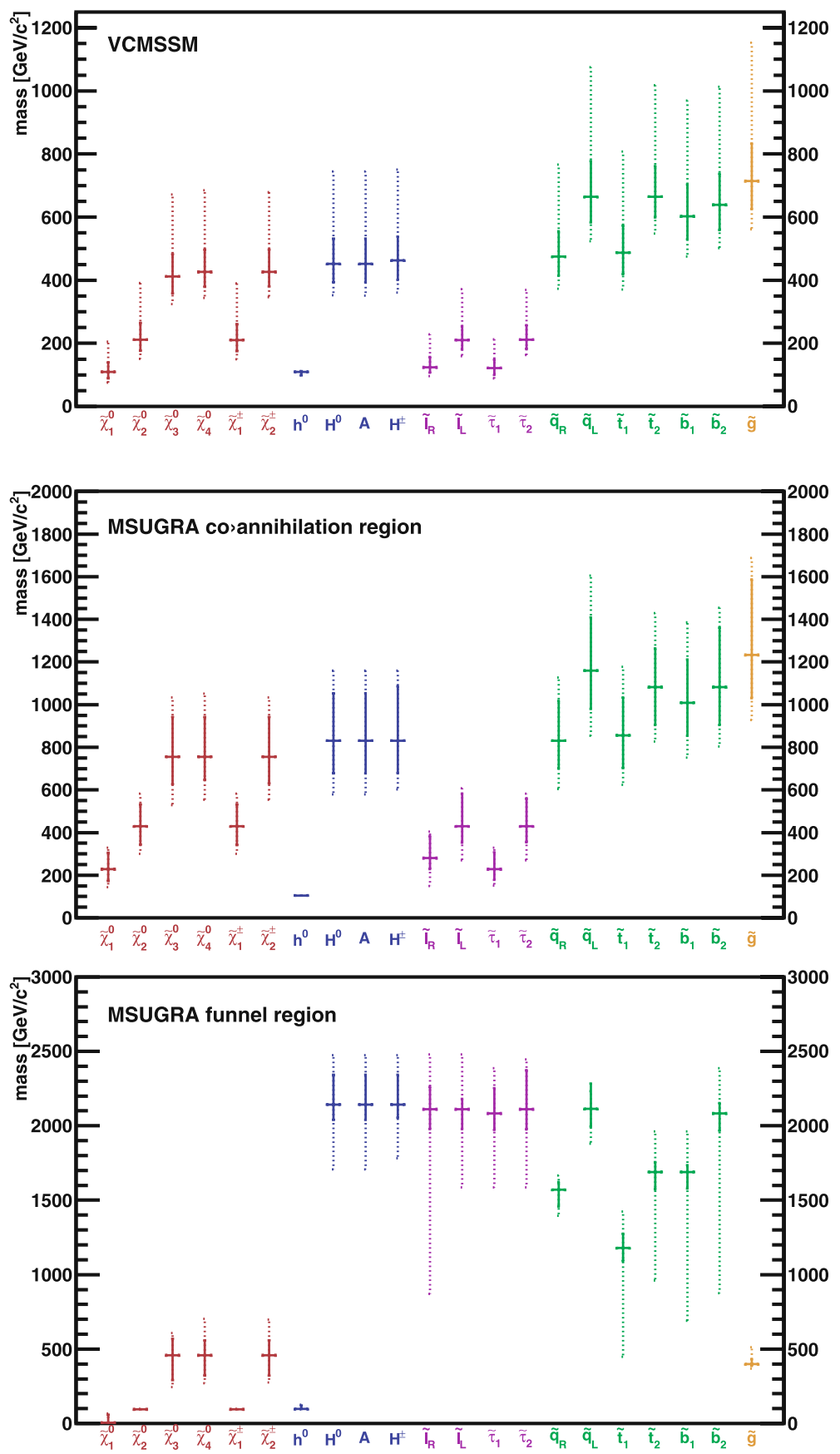

(more probable, but still disfavoured scenario compared to VCMSSM, CMSSM and NUHM1) coannihilation region of mSUGRA, where the best fit has a gluino mass around 1200 to $1400 \mathrm{GeV}$.

The bimodality of the mSUGRA likelihood function is reflected again in the predictions for $m_{\tilde{q}_{R}}-m_{\tilde{g}}$ (middle left) and $m_{\tilde{\tau}_{1}}-m_{\tilde{\chi}_{1}^{0}}$ (middle right) in Fig. 6. The two different mSUGRA minima yield different signs for $m_{\tilde{q}_{R}}-m_{\tilde{g}}$, whereas $m_{\tilde{g}}>m_{\tilde{q}_{R}}$ is favoured in the VCMSSM, as was shown previously to be favoured in the CMSSM [81, 82], and to a rather lesser extent in the NUHM1 [81, 82]. Thus, the sign and magnitude of $m_{\tilde{q}_{R}}-m_{\tilde{g}}$ are potential diagnostic tools for discriminating between different models. ${ }^{9}$ We note also the different predictions for $m_{\tilde{\tau}_{1}}-m_{\tilde{\chi}_{1}^{0}}$ the VCMSSM

${ }^{9}$ We recall that dijet + missing energy events due to the decay chain $\tilde{g} \rightarrow \tilde{q}_{R}+\bar{q}, \tilde{q}_{R} \rightarrow q+\tilde{\chi}_{1}^{0}$ are in general favoured when $m_{\tilde{q}_{R}}<m_{\tilde{g}}$, whereas there are expected to be a larger fraction of four-jet + missing energy events when $m_{\tilde{q}_{R}}>m_{\tilde{g}}$, and different decay chains occur via $\tilde{q}_{L}, \tilde{t}_{1,2}$ and $\tilde{b}_{1,2}$. 
羌

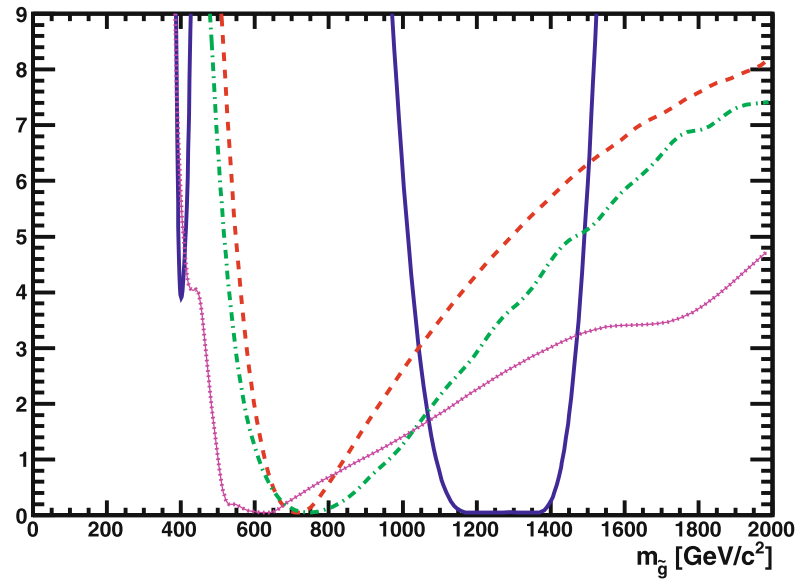

ॠ

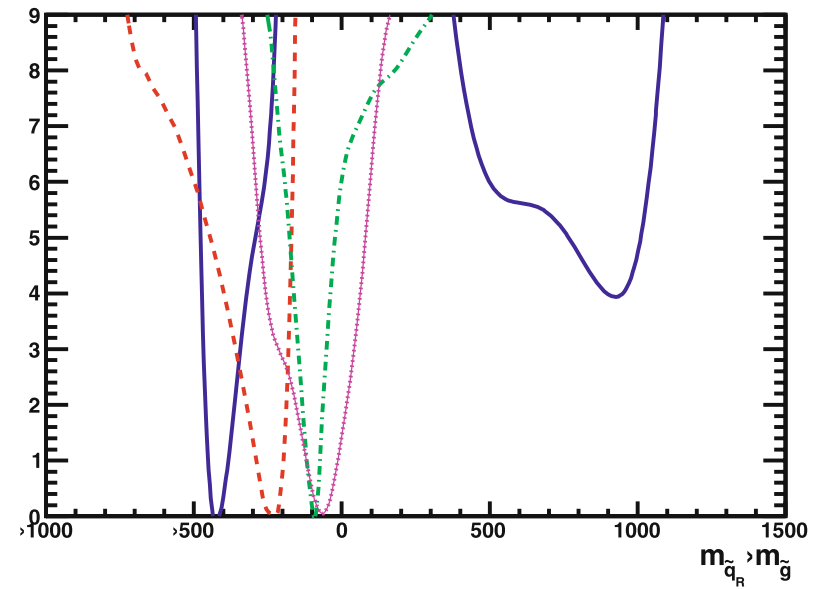

$\stackrel{2}{\dddot{4}}$

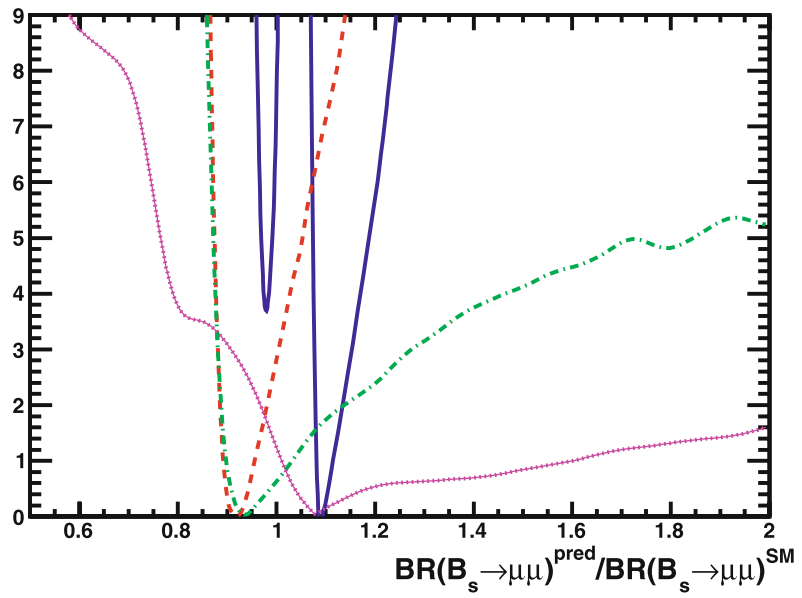

Fig. 6 (Color online) The likelihood functions for (top) $m_{\tilde{g}}$ and $m_{\tilde{\chi}_{1}^{0}}$, (middle) $m_{\tilde{q}_{R}}-m_{\tilde{g}}$ and $m_{\tilde{\tau}_{1}}-m_{\tilde{\chi}_{1}^{0}}$, and (bottom) $\operatorname{BR}\left(B_{s} \rightarrow \mu^{+} \mu^{-}\right)$ (normalized to the SM estimate) and $\sigma_{p}^{\text {SI }}$. In each case, we compare the

predicts a very small mass difference, as was shown previously in the CMSSM, and so does mSUGRA in the coannihilation region. However, in the rapid-annihilation region mSUGRA predicts mass differences that may be large, as was previously shown to be possible (to a lesser extent) in
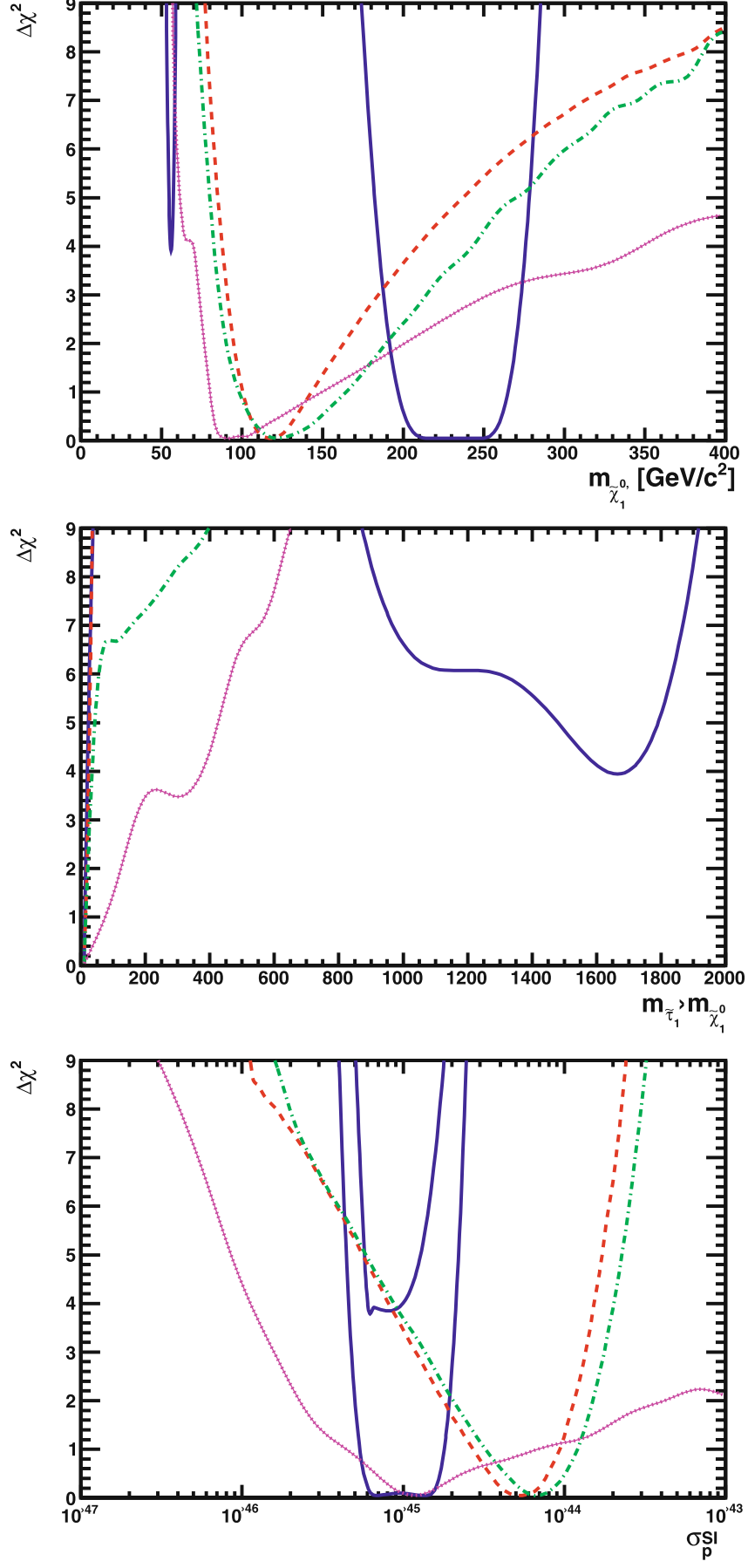

predictions in MSUGRA (blue solid line), the VCMSSM (red dashed line), the CMSSM (green dash-dotted line) and the NUHM1 (purple dotted line)

the NUHM1 [82]. Thus discovery of a light gluino at the LHC and/or a light neutralino LSP would not necessarily imply that the lightest slepton would have a mass close to that of the LSP, and the lighter stau could be too heavy for an $e^{+} e^{-}$collider with $\sqrt{s}=1 \mathrm{TeV}$. However, in this sce- 


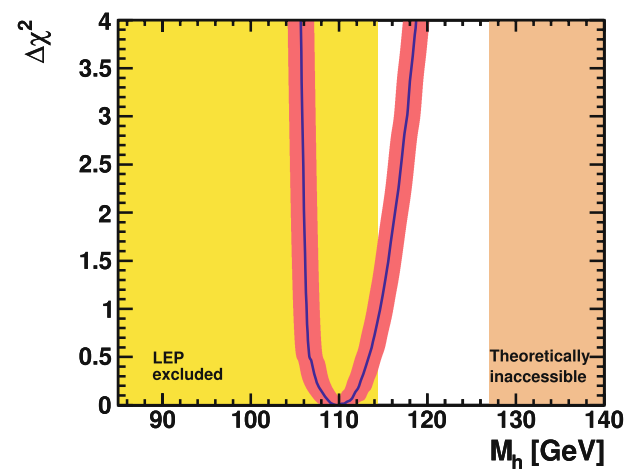

Fig. 7 (Color online) The $\chi^{2}$ functions for $M_{h}$ in the VCMSSM (left) and mSUGRA (right), including the theoretical uncertainties (red bands), as well as the SM prediction for $M_{h}$ based on a precision electroweak fit (blue band). Also shown is the mass range for a SM-like Higgs boson excluded by LEP (yellow shading), and

nario the whole chargino and neutralino spectrum would be accessible at an $e^{+} e^{-}$collider with $\sqrt{s}=1 \mathrm{TeV}$, see Fig. 5.

We see in the bottom left panel of Fig. 6 that in the $\operatorname{VCMSSM}$ a value of $\operatorname{BR}\left(B_{s} \rightarrow \mu^{+} \mu^{-}\right)$slightly lower than the SM value is favoured, although larger values cannot be excluded. On the other hand, in mSUGRA in the coannihilation region, with its relatively large values of $\tan \beta$, we find a preferred value of $\operatorname{BR}\left(B_{s} \rightarrow \mu^{+} \mu^{-}\right)$that is slightly larger than in the SM, whereas a range around the SM level is favoured in the light Higgs funnel region. As already seen in [82], the minimum of $\chi^{2}$ in the NUHM1 is at a somewhat higher value of $\operatorname{BR}\left(B_{S} \rightarrow \mu^{+} \mu^{-}\right)$than in the SM, whereas the best-fit value in the CMSSM is again slightly smaller, albeit with considerable uncertainty. The values below the SM prediction arise from chargino-induced $Z b s$ penguin diagrams, that yield analogous but smaller reductions in $\operatorname{BR}(K \rightarrow \pi v \bar{v})$. However, the $\mathrm{SM}$ prediction has an uncertainty of about $10 \%$, and $\mathcal{O}(100) B_{s} \rightarrow \mu^{+} \mu^{-}$decays would be needed to match this error, so the differences between the mSUGRA, VCMSSM and best-fit CMSSM predictions are probably unobservable.

Turning to the likelihood functions for $\sigma_{p}^{\mathrm{SI}}$ in the bottom right panel of Fig. 6 (calculated assuming a $\pi-\mathrm{N}$ scattering $\sigma$ term $\Sigma_{N}=64 \mathrm{MeV}$ : see, e.g., [116] for a discussion of the implications of modifying this assumption) we see that a range between $10^{-45}$ and $10^{-44} \mathrm{~cm}^{2}$ is favoured in the VCMSSM, whereas in mSUGRA a range between $2 \times 10^{-46} \mathrm{~cm}^{2}$ and $5 \times 10^{-45} \mathrm{~cm}^{2}$ is favoured.

Finally, in Fig. 7 we display the one-dimensional $\chi^{2}$ likelihood functions for $M_{h}$ in the VCMSSM (left) and mSUGRA (right), not including the direct limits from LEP and the Tevatron. ${ }^{10}$ For each model we display the like-

${ }^{10}$ See [80-82] for the corresponding plots in the CMSSM and NUHM1.

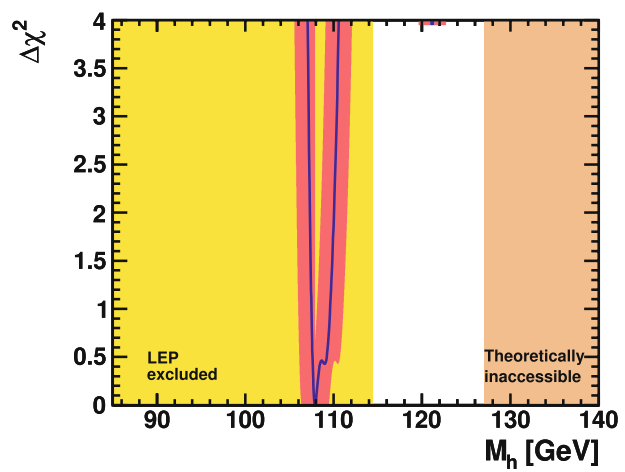

the mass range that is theoretically inaccessible in TeV-scale SUSY (beige shading). Note in right panel the secondary minimum at $M_{h}=121.1 \mathrm{GeV}, \Delta \chi^{2}=3.9$ and the accompanying narrow horizontal red band

lihood functions, including the theoretical uncertainties (red bands), which we take to be $1.5 \mathrm{GeV}$ in all models. For comparison, we also show the mass range excluded for a SM-like Higgs boson (yellow shading) obtained at LEP $[114,115]$. This limit is valid since the VCMSSM and mSUGRA are sub-spaces of the more general CMSSM parameter space, where the LEP limits have been shown to be valid [117, 118]. Values somewhat below the LEP exclusion are favoured in the coannihilation region of mSUGRA, in the VCMSSM and the CMSSM, entailing $\chi^{2}$ prices of 3.9 (1.1) (1.4), as discussed previously. In the mSUGRA coannihilation case, the global minimum of $\chi^{2}$ found when the LEP constraint is disregarded is in an isolated region at low $\left(m_{0}, m_{1 / 2}\right)$. When the LEP Higgs constraint is applied, this region is strongly disfavoured, and the global minimum moves to the green star shown in Fig. 1, located at much larger $\left(m_{0}, m_{1 / 2}\right)$ and with $\Delta \chi^{2}=3.9$. This other minimum is reflected in the blue point at $M_{h}=121.1 \mathrm{GeV}$ with $\Delta \chi^{2}=3.9$ and the corresponding horizontal red line visible at the top of the right panel in Fig. 7. Comparably large values of $M_{h} \sim 117,120 \mathrm{GeV}$ are favoured in the funnel region of mSUGRA and in the NUHM1, so these models are naturally consistent with the LEP bound on a SM-like Higgs boson.

\section{Conclusions}

We have completed in this paper the frequentist analysis of a nested sequence of variants of the MSSM: NUHM1 $\ni$ CMSSM $\ni$ VCMSSM $\ni$ mSUGRA, discussing in each case the best-fit point, the minimum $\chi^{2} /$ dof, the 68 and $95 \% \mathrm{CL}$ regions and aspects of the favoured ranges of particle masses and other observables. We found previously that the restriction from the NUHM1 to the CMSSM does not change drastically the position of the best-fit point or the favoured 
ranges of parameters and observables. This reflects the fact that the present data do not constrain significantly the heavier Higgs bosons of the MSSM, and so there is no significant tension in the CMSSM fit arising from that sector. Likewise, we found in the present paper that the restriction from the CMSSM to the VCMSSM does not have a large impact on the position of the best-fit point or on the favoured ranges. This reflects the fact that the present data also do not constrain significantly the trilinear soft SUSY-breaking parameter $A_{0}$, and so there is no significant $\chi^{2}$ price to be paid when setting $A_{0}=B_{0}+m_{0}$ as in the VCMSSM. Fits in all three of the NUHM1, CMSSM and VCMSSM frameworks have good absolute probabilities in our frequentist analyses.

On the other hand, in mSUGRA two almost equally good best-fit points coexist, with rather different MSSM parameter values, significantly higher values of $\chi^{2}$ and lower absolute probability, somewhat disfavouring this scenario. This reflects the fact that the neutralino LSP constraint $m_{\tilde{\chi}_{1}^{0}}<m_{3 / 2}$ excludes the best fit found in the VCMSSM and forces instead either rather larger values of $m_{0}, m_{1 / 2}$ and $\tan \beta$ or points along the narrow light Higgs rapidannihilation funnel with small $m_{1 / 2}$ but large $m_{0}$.

In each of the NUHM1, CMSSM and VCMSSM there is a significant chance of observing first hints of SUSY in the 2011/2012 run of the LHC, whereas this may be more problematic in mSUGRA, depending on whether its parameters lie in the rapid-annihilation funnel or in the higher-mass coannihilation region. Correspondingly, early hints of SUSY at the LHC in this first run might further favour the NUHM1, CMSSM and VCMSSM frameworks over the higher-mass mSUGRA option in the coannihilation region. In the rapidannihilation funnel region of mSUGRA one would expect abundant production of gluinos, whereas the first appearances of squarks, having masses in excess of $1 \mathrm{TeV}$, would only happen at a somewhat later stage. If no sign of SUSY particles shows up in the early LHC run, in particular if the integrated luminosity (and energy) goes significantly beyond the currently foreseen $1 / \mathrm{fb}$ at $7 \mathrm{TeV}$, the scenarios predicting a relatively low SUSY scale could soon come under pressure. At the time of writing, the ATLAS and CMS collaborations are each examining some $35 / \mathrm{pb}$ of analysable data.

Acknowledgements We thank Gino Isidori for valuable discussions. Work supported in part by the European Community's Marie-Curie Research Training Network under contract MRTN-CT-2006-035505 'Tools and Precision Calculations for Physics Discoveries at Colliders'. The work of KAO was supported in part by DOE grant DE-FG0294ER-40823 at the University of Minnesota. The work of S.H. was supported by the Spanish MICINN's Consolider-Ingenio 2010 Programme under grant MultiDark CSD2009-00064.

Open Access This article is distributed under the terms of the Creative Commons Attribution Noncommercial License which permits any noncommercial use, distribution, and reproduction in any medium, provided the original author(s) and source are credited.
Note Added in Proof Since the submission of this paper, the first results of searches for supersymmetry have been published by the CMS and ATLAS Collaborations $[119,120]$. These change somewhat the parameters of the best-fit points and the $68 \%$ and $95 \%$ CL regions in the models studied here, but do not disfavour any of them strongly [121].

\section{References}

1. L. Maiani, in Proceedings of the Gif-sur-Yvette Summer School on Particle Physics (1979), pp. 1-52

2. G. 't Hooft, in Recent developments in Gauge Theories. Proceedings of the NATO Advanced Study Institute, Cargèse, 1979, ed. by G. 't Hooft et al. (Plenum Press, New York, 1980)

3. E. Witten, Phys. Lett. B 105, 267 (1981)

4. J.R. Ellis, S. Kelley, D.V. Nanopoulos, Phys. Lett. B 249, 441 (1990)

5. J.R. Ellis, S. Kelley, D.V. Nanopoulos, Phys. Lett. B 260, 131 (1991)

6. U. Amaldi, W. de Boer, H. Furstenau, Phys. Lett. B 260, 447 (1991)

7. C. Giunti, C.W. Kim, U.W. Lee, Mod. Phys. Lett. A 6, 1745 (1991)

8. J. Ellis, J.S. Hagelin, D.V. Nanopoulos, K.A. Olive, M. Srednicki, Nucl. Phys. B 238, 453 (1984)

9. H. Goldberg, Phys. Rev. Lett. 50, 1419 (1983)

10. H. Pagels, J. Primack, Phys. Rev. Lett. 48, 223 (1982)

11. S. Weinberg, Phys. Rev. Lett. 48, 1303 (1982)

12. J. Ellis, A.D. Linde, D.V. Nanopoulos, Phys. Lett. 118, 59 (1982)

13. D.V. Nanopoulos, K.A. Olive, M. Srednicki, Phys. Lett. B 127, $30(1983)$

14. L.M. Krauss, Nucl. Phys. B 227, 55 (1983)

15. M.Y. Khlopov, A.D. Linde, Phys. Lett. B 138, 265 (1984)

16. J.R. Ellis, K.A. Olive, Y. Santoso, V.C. Spanos, Phys. Lett. B 588, 7 (2004). arXiv:hep-ph/0312262

17. J.L. Feng, A. Rajaraman, F. Takayama, Phys. Rev. Lett. 91, 011302 (2003). arXiv:hep-ph/0302215

18. J.L. Feng, S. Su, F. Takayama, Phys. Rev. D 70, 075019 (2004). arXiv:hep-ph/0404231

19. J.R. Ellis, G. Ridolfi, F. Zwirner, Phys. Lett. B 257, 83 (1991)

20. J.R. Ellis, G. Ridolfi, F. Zwirner, Phys. Lett. B 262, 477 (1991)

21. Y. Okada, M. Yamaguchi, T. Yanagida, Phys. Lett. B 262, 54 (1991)

22. Y. Okada, M. Yamaguchi, T. Yanagida, Prog. Theor. Phys. 85, 1 (1991)

23. A. Yamada, Phys. Lett. B 263, 233 (1991)

24. H.E. Haber, R. Hempfling, Phys. Rev. Lett. 66, 1815 (1991)

25. M. Drees, M.M. Nojiri, Phys. Rev. D 45, 2482 (1992)

26. P.H. Chankowski, S. Pokorski, J. Rosiek, Phys. Lett. B 274, 191 (1992)

27. P.H. Chankowski, S. Pokorski, J. Rosiek, Phys. Lett. B 286, 307 (1992)

28. G. Degrassi, S. Heinemeyer, W. Hollik, P. Slavich, G. Weiglein, Eur. Phys. J. C 28, 133 (2003). arXiv:hep-ph/0212020

29. The Muon g-2 Collaboration, Phys. Rev. Lett. 92, 161802 (2004). arXiv:hep-ex/0401008

30. G. Bennett et al. (The Muon g-2 Collaboration), Phys. Rev. D 73, 072003 (2006). arXiv:hep-ex/0602035

31. D. Stockinger, J. Phys. G 34, R45 (2007). arXiv:hep-ph/0609168

32. J. Miller, E. de Rafael, B. Roberts, Rep. Prog. Phys. 70, 795 (2007). arXiv:hep-ph/0703049

33. J. Prades, E. de Rafael, A. Vainshtein, arXiv:0901.0306 [hep-ph]

34. F. Jegerlehner, A. Nyffeler, Phys. Rep. 477, 1 (2009). arXiv: 0902.3360 [hep-ph]

35. M. Davier, A. Hoecker, B. Malaescu, C.Z. Yuan, Z. Zhang, Eur. Phys. J. C 66, 1 (2010). arXiv:0908.4300 [hep-ph] 
36. J. Prades, Acta Phys. Pol. Suppl. 3, 75 (2010). arXiv:0909.2546 [hep-ph]

37. T. Teubner, K. Hagiwara, R. Liao, A.D. Martin, D. Nomura, arXiv:1001.5401 [hep-ph]

38. M. Davier, A. Hoecker, B. Malaescu, Z. Zhang, arXiv:1010.4180 [hep-ph]

39. H.E. Haber, G.L. Kane, Phys. Rep. 117, 75 (1985)

40. M. Drees, M.M. Nojiri, Phys. Rev. D 47, 376 (1993). arXiv: hep-ph/9207234

41. H. Baer, M. Brhlik, Phys. Rev. D 53, 597 (1996). arXiv:hep-ph/ 9508321

42. H. Baer, M. Brhlik, Phys. Rev. D 57, 567 (1998). arXiv:hep-ph/ 9706509

43. J.R. Ellis, T. Falk, K.A. Olive, M. Schmitt, Phys. Lett. B 388, 97 (1996). arXiv:hep-ph/9607292

44. J.R. Ellis, T. Falk, K.A. Olive, M. Schmitt, Phys. Lett. B 413, 355 (1997). arXiv:hep-ph/9705444

45. J.R. Ellis, T. Falk, G. Ganis, K.A. Olive, M. Schmitt, Phys. Rev. D 58, 095002 (1998). arXiv:hep-ph/9801445

46. V.D. Barger, C. Kao, Phys. Rev. D 57, 3131 (1998). arXiv: hep-ph/9704403

47. J.R. Ellis, T. Falk, G. Ganis, K.A. Olive, Phys. Rev. D 62, 075010 (2000). arXiv:hep-ph/0004169

48. J.R. Ellis, T. Falk, G. Ganis, K.A. Olive, M. Srednicki, Phys. Lett. B 510, 236 (2001). arXiv:hep-ph/0102098

49. V.D. Barger, C. Kao, Phys. Lett. B 518, 117 (2001). arXiv: hep-ph/0106189

50. L. Roszkowski, R. Ruiz de Austri, T. Nihei, J. High Energy Phys. 0108, 024 (2001). arXiv:hep-ph/0106334

51. A. Djouadi, M. Drees, J.L. Kneur, J. High Energy Phys. 0108, 055 (2001). arXiv:hep-ph/0107316

52. U. Chattopadhyay, A. Corsetti, P. Nath, Phys. Rev. D 66, 035003 (2002). arXiv:hep-ph/0201001

53. J.R. Ellis, K.A. Olive, Y. Santoso, New J. Phys. 4, 32 (2002). arXiv:hep-ph/0202110

54. H. Baer, C. Balazs, A. Belyaev, J.K. Mizukoshi, X. Tata, Y. Wang, J. High Energy Phys. 0207, 050 (2002). arXiv:hep-ph/ 0205325

55. R. Arnowitt, B. Dutta, arXiv:hep-ph/0211417

56. J.R. Ellis, K.A. Olive, Y. Santoso, V.C. Spanos, Phys. Lett. B 565, 176 (2003). arXiv:hep-ph/0303043

57. H. Baer, C. Balazs, J. Cosmol. Astropart. Phys. 0305, 006 (2003). arXiv:hep-ph/0303114

58. A.B. Lahanas, D.V. Nanopoulos, Phys. Lett. B 568, 55 (2003). arXiv:hep-ph/0303130

59. U. Chattopadhyay, A. Corsetti, P. Nath, Phys. Rev. D 68, 035005 (2003). arXiv:hep-ph/0303201

60. C. Munoz, Int. J. Mod. Phys. A 19, 3093 (2004). arXiv:hep-ph/ 0309346

61. J.R. Ellis, K.A. Olive, Y. Santoso, V.C. Spanos, Phys. Rev. D 69, 095004 (2004). arXiv:hep-ph/0310356

62. J. Ellis, S. Heinemeyer, K. Olive, G. Weiglein, J. High Energy Phys. 0502, 013 hep-ph/0411216

63. J.R. Ellis, S. Heinemeyer, K.A. Olive, G. Weiglein, J. High Energy Phys. 0605, 005 (2006). arXiv:hep-ph/0602220

64. H. Baer, A. Mustafayev, S. Profumo, A. Belyaev, X. Tata, Phys. Rev. D 71, 095008 (2005). arXiv:hep-ph/0412059

65. H. Baer, A. Mustafayev, S. Profumo, A. Belyaev, X. Tata, J. High Energy Phys. 0507, 065 (2005). hep-ph/0504001

66. J.R. Ellis, K.A. Olive, P. Sandick, Phys. Rev. D 78, 075012 (2008). arXiv:0805.2343 [hep-ph]

67. J. Polonyi, Generalization of the Massive Scalar Multiplet Coupling to the Supergravity, Hungary Central Inst Res-KFKI-7793

68. E. Cremmer, B. Julia, J. Scherk, P. van Nieuwenhuizen, S. Ferrara, L. Girardello, Phys. Lett. B 79, 231 (1978)
69. E. Cremmer, B. Julia, J. Scherk, S. Ferrara, L. Girardello, P. van Nieuwenhuizen, Nucl. Phys. B 147, 105 (1979)

70. R. Barbieri, S. Ferrara, C.A. Savoy, Phys. Lett. B 119, 343 (1982)

71. A.H. Chamseddine, R.L. Arnowitt, P. Nath, Phys. Rev. Lett. 49, 970 (1982)

72. For reviews, see: H.P. Nilles, Phys. Rep. 110, 1 (1984)

73. A. Brignole, L.E. Ibanez, C. Munoz, in Perspectives on supersymmetry, ed. by G.L. Kane, pp. 125-148. arXiv:hep-ph/ 9707209

74. L.E. Ibanez, G.G. Ross, Phys. Lett. B 110, 215 (1982)

75. L.E. Ibanez, Phys. Lett. B 118, 73 (1982)

76. J.R. Ellis, D.V. Nanopoulos, K. Tamvakis, Phys. Lett. B 121, 123 (1983)

77. L. Alvarez-Gaume, J. Polchinski, M.B. Wise, Nucl. Phys. B 221, $495(1983)$

78. J.R. Ellis, K.A. Olive, Y. Santoso, V.C. Spanos, Phys. Lett. B 573, 162 (2003). arXiv:hep-ph/0305212

79. J.R. Ellis, K.A. Olive, Y. Santoso, V.C. Spanos, Phys. Rev. D 70, 055005 (2004). arXiv:hep-ph/0405110

80. O. Buchmueller et al., Phys. Lett. B 657, 87 (2007). arXiv: 0707.3447 [hep-ph]

81. O. Buchmueller et al., J. High Energy Phys. 0809, 117 (2008). arXiv:0808.4128 [hep-ph]

82. O. Buchmueller et al., Eur. Phys. J. C 64, 391 (2009). arXiv: 0907.5568 [hep-ph]

83. O. Buchmueller et al., Phys. Rev. D 81, 035009 (2010). arXiv:0912.1036 [hep-ph]

84. R.H. Cyburt, J.R. Ellis, B.D. Fields, K.A. Olive, V.C. Spanos, J. Cosmol. Astropart. Phys. 0611, 014 (2006). arXiv:astro-ph/ 0608562

85. J.R. Ellis, K.A. Olive, E. Vangioni, Phys. Lett. B 619, 30 (2005). arXiv:astro-ph/0503023

86. K. Jedamzik, K.Y. Choi, L. Roszkowski, R. Ruiz de Austri, J. Cosmol. Astropart. Phys. 0607, 007 (2006). arXiv:hep-ph/ 0512044

87. M. Kusakabe, T. Kajino, R.N. Boyd, T. Yoshida, G.J. Mathews, Phys. Rev. D 76, 121302 (2007). arXiv:0711.3854 [astro-ph]

88. T. Jittoh, K. Kohri, M. Koike, J. Sato, T. Shimomura, M. Yamanaka, Phys. Rev. D 76, 125023 (2007). arXiv:0704.2914 [hep$\mathrm{ph}]$

89. M. Pospelov, J. Pradler, F.D. Steffen, J. Cosmol. Astropart. Phys. 0811, 020 (2008). arXiv:0807.4287 [hep-ph]

90. G.B. Gelmini, Nucl. Phys. B, Proc. Suppl. 194, 63 (2009). arXiv: 0907.1694 [hep-ph]

91. A. Dedes, A.B. Lahanas, K. Tamvakis, Phys. Rev. D 53, 3793 (1996). arXiv:hep-ph/9504239

92. B.C. Allanach, Comput. Phys. Commun. 143, 305 (2002). arXiv: hep-ph/0104145

93. S. Heinemeyer, W. Hollik, G. Weiglein, Eur. Phys. J. C 9, 343 (1999). arXiv:hep-ph/9812472

94. S. Heinemeyer, W. Hollik, G. Weiglein, Comput. Phys. Commun. 124, 76 (2000). arXiv:hep-ph/9812320. See http://www. feynhiggs.de

95. M. Frank, T. Hahn, S. Heinemeyer, W. Hollik, H. Rzehak, G. Weiglein, J. High Energy Phys. 0702, 047 (2007). arXiv: hep-ph/0611326

96. T. Moroi, Phys. Rev. D 53, 6565 (1996). Erratum-ibid. D 56, 4424 (1997). arXiv:hep-ph/9512396

97. G. Degrassi, G.F. Giudice, Phys. Rev. D 58, 053007 (1998). arXiv:hep-ph/9803384

98. S. Heinemeyer, D. Stockinger, G. Weiglein, Nucl. Phys. B 690, 62 (2004). arXiv:hep-ph/0312264

99. S. Heinemeyer, D. Stockinger, G. Weiglein, Nucl. Phys. B 699, 103 (2004). arXiv:hep-ph/0405255

100. G. Isidori, P. Paradisi, Phys. Lett. B 639, 499 (2006). arXiv: hep-ph/0605012 
101. G. Isidori, F. Mescia, P. Paradisi, D. Temes, Phys. Rev. D 75, 115019 (2007). arXiv:hep-ph/0703035, and references therein

102. F. Mahmoudi, Comput. Phys. Commun. 178, 745 (2008). arXiv:0710.2067 [hep-ph] and arXiv:0808.3144 [hep-ph]

103. D. Eriksson, F. Mahmoudi, O. Stal, J. High Energy Phys. 0811, 035 (2008). arXiv:0808.3551 [hep-ph]

104. S. Heinemeyer, W. Hollik, D. Stockinger, A.M. Weber, G. Weiglein, J. High Energy Phys. 0608, 052 (2006). arXiv:hep-ph/ 0604147

105. S. Heinemeyer, W. Hollik, A.M. Weber, G. Weiglein, J. High Energy Phys. 0804, 039 (2008). arXiv:0710.2972 [hep-ph]

106. G. Belanger, F. Boudjema, A. Pukhov, A. Semenov, Comput. Phys. Commun. 176, 367 (2007). arXiv:hep-ph/0607059

107. G. Belanger, F. Boudjema, A. Pukhov, A. Semenov, Comput. Phys. Commun. 149, 103 (2002). arXiv:hep-ph/0112278

108. G. Belanger, F. Boudjema, A. Pukhov, A. Semenov, Comput. Phys. Commun. 174, 577 (2006). arXiv:hep-ph/0405253

109. P. Gondolo, J. Edsjo, P. Ullio, L. Bergstrom, M. Schelke, E.A. Baltz, New Astron. Rev. 49, 149 (2005)

110. P. Gondolo, J. Edsjo, P. Ullio, L. Bergstrom, M. Schelke, E.A. Baltz, J. Cosmol. Astropart. Phys. 0407, 008 (2004). arXiv: astro-ph/0406204
111. P. Skands et al., J. High Energy Phys. 0407, 036 (2004). arXiv:hep-ph/0311123

112. B. Allanach et al., Comput. Phys. Commun. 180, 8 (2009). arXiv:0801.0045 [hep-ph]

113. E. Komatsu et al., arXiv:1001.4538 [astro-ph.CO]

114. R. Barate et al. (ALEPH, DELPHI, L3, OPAL Collaborations and LEP Working Group for Higgs boson searches), Phys. Lett. B 565, 61 (2003). arXiv:hep-ex/0306033

115. S. Schael et al. (ALEPH, DELPHI, L3, OPAL Collaborations and LEP Working Group for Higgs boson searches), Eur. Phys. J. C 47, 547 (2006). arXiv:hep-ex/0602042

116. J.R. Ellis, K.A. Olive, C. Savage, Phys. Rev. D 77, 065026 (2008). arXiv:0801.3656 [hep-ph]

117. J.R. Ellis, S. Heinemeyer, K.A. Olive, G. Weiglein, Phys. Lett. B 515, 348 (2001). arXiv:hep-ph/0105061

118. S. Ambrosanio, A. Dedes, S. Heinemeyer, S. Su, G. Weiglein, Nucl. Phys. B 624, 3 (2002). arXiv:hep-ph/0106255

119. V. Khachatryan et al. (CMS Collaboration), arXiv:1101.1628 [hep-ex]

120. G. Aad et al. (ATLAS Collaboration), arXiv:1102.2357 [hep-ex]

121. O. Buchmueller et al., arXiv:1102.4585 [hep-ph] 